\title{
Dynamic experience of architectural forms affects arousal and valence perception in virtual environments
}

\section{Paolo Presti}

Department of Medicine and Surgery, University of Parma

\section{Davide Ruzzon}

TUNED, Lombardini22

\section{Pietro Avanzini}

Institute of Neuroscience, National Research Council of Italy

\section{Fausto Caruana}

Institute of Neuroscience, National Research Council of Italy

Giacomo Rizzolatti

Institute of Neuroscience, National Research Council of Italy

Giovanni Vecchiato ( $\nabla$ giovanni.vecchiato@in.cnr.it)

Institute of Neuroscience, National Research Council of Italy

\section{Research Article}

Keywords: pleasant and unpleasant, Comitato Etico AVEN, Ceiling, SideWalls, constant, increasing

Posted Date: September 21st, 2021

DOI: https://doi.org/10.21203/rs.3.rs-910384/v1

License: (1) This work is licensed under a Creative Commons Attribution 4.0 International License. Read Full License 


\section{Abstract}

The built environment represents the stage surrounding our everyday life activities. To investigate the impact that architectural features have on individuals' affective states, we measured their judgments of perceived valence (pleasant and unpleasant) and arousal after the dynamic experience of a progressive change of the environment within virtual decontextualized spaces. To this aim, we developed a parametric model which allowed to create 54 virtual architectural spaces characterized by a progressive change of side walls distance, ceiling and windows height as well as colour of the environment.

Decreasing side walls distance, ceiling height variation as well as increasing windows height significantly affected the emotional state of the participants within virtual environments. Indeed, such architectural spaces generated high arousing and unpleasant states according to subjective judgement. Overall, we observed that valence and arousal scores are affected by all the dynamic form factors which modulated the spaciousness of the surrounding. Showing that virtual reality enables the possibility to measure the emotional impact of pure and decontextualized architectural features, we contribute to foster a humancentered approach to design where mental state of people is fundamental for the creation of novel spaces promoting wellbeing.

\section{Introduction}

A crucial but largely unexplored issue of human experience concerns how affective states are influenced by the dynamical change of spatial features when walking through a built environment. Previous studies using static 2D representations showed that several architectural features massively impact on the observer affective states, typically measured in terms of valence and arousal ${ }^{1}$.

Valence represents the extent to which an architectural space makes an occupant feel good or bad. In recent studies participants reported higher beautiful judgements for 2D representations of architectures with high ceiling and open spaces ${ }^{2,3}$, which were also perceived as more pleasant ${ }^{4}$. The presence of windows is also typically linked to pleasant sensation ${ }^{5}$, since they enable an outdoor view and create a more spacious perception of the environment ${ }^{6,7}$. Moreover, cold colours receive typically higher valence ratings with respect to warm ones, thus moving people preferences towards cold environments ${ }^{8-10}$.

The arousal level can be modulated by the amount of light penetrating through the windows, which promote circadian rhythms and contribute to the overall well-being of people, diminishing their stress level ${ }^{11}$. Furthermore, cold coloured environments were associated with a peaceful sensation, while warm colours generated higher arousing states ${ }^{10,12}$. Previous research also reported that enclosed spaces increase vulnerability to stress and prolong the occupant's stress response ${ }^{13}$, while spaces with low ceiling were found to generate a sense of confinement, thus being associated to higher arousing states 14,15 .

However, the study mentioned above used everyday-life architectural spaces in specific context such as living rooms, meeting halls, hotel receptions, etc., each carrying a particular semantic content. These 
contextual factors might affect the subjective perception of specific architectural factors. Moreover, evidence collected during the perception of static virtual environments ${ }^{16-18}$ and those with $2 \mathrm{D}$ representations confounded the whole architectural experience making it far from being realistic.

To overcome the present limitations, we conceived virtual environments without any element of interior design to deprive the space of its context. These were perceived as a virtual promenade to allow the dynamic experience of the architectural features as a real-life situation. Virtual reality technologies ensured a highly immersive experience for the participants as if they were physically situated in real architectures ${ }^{19}$. We built a full-factorial design of 54 virtual environments manipulating the variation of architectural elements such as the sidewall distance, ceiling height, windows height and colour. This is to demonstrate that the dynamic experience of decontextualized architectural forms manipulates affective states through a progressive modulation of spaciousness, as previously suggested ${ }^{20,21}$.

\section{Material And Methods}

\section{Participants}

Twenty-nine subjects were recruited for this experiment ( $27.35 \pm 3.7$ years, 16 female). The sample size was determined using a power analysis computed through the $\mathrm{G}^{*}$ Power software ${ }^{22}$, considering the $2 \times 3 \times 3 \times 3$ within subject design with the "as in SPSS" option and setting the significance level (a) at 0.05 , the desired power $(1-\beta)$ to 0.95 , the number of groups to 1 , the number of repetition to 54 and the nonsphericity correction $\nabla$ to 1 . The value of the $\eta_{p}{ }^{2}$ was set to 0.03 based on previous research ${ }^{4}$. All subjects reported normal or corrected-to-normal vision and no previous history of neurological disorders. The study was approved by the local ethical committee (Comitato Etico AVEN) and conducted according to the principles expressed in the Declaration of Helsinki. Each participant provided written informed consent before participating in the experiment.

\section{Stimuli}

A 3D virtual architecture was generated as a combination of three consecutive nuclei of fixed dimension: $4 \mathrm{~m} \times 4 \mathrm{~m} \times 7 \mathrm{~m}$ (width $\mathrm{x}$ height $\times$ length) with $1 \mathrm{~m} \times 6.8 \mathrm{~m}$ (height $\mathrm{x}$ length) windows located in the middle of the side walls for each nucleus. A parametric model was then designed on Grasshopper (https://www.rhino3d.com/6/new/grasshopper/) and then imported on the online platform ShapeDiver (https://shapediver.com/) to generate 27 different architectures varying architectural factors such as the distance between the side walls (SideWalls), the height of the ceiling (Ceiling) and the height of the windows (Windows). Specifically, the parametric model allowed to increase, decrease and keep constant the architectural factors between one nucleus and the next one. Thus, we realized variations of $\pm 0.8 \mathrm{~m}$ for the factors SideWalls and Ceiling. The height of the windows changed as a percentage of the ceiling height: $75 \%$ and $100 \%$ of the ceiling height in the second and third nucleus respectively when increasing, $25 \%$ and $0 \%$ when decreasing, $50 \%$ when constant. All the virtual architectures were endowed with two doors: an opened one in the first nucleus as entrance and a closed one at the end of the third nucleus. 
Such configuration enhanced the realism of the dynamic experience of the architecture, giving the subject the impression of being inside an environment suitable for a promenade, providing an entry and an exit point. The 3D architectures generated in Shapediver were imported into Unity (2019.1.0f2, https://unity.com/). Here, to transform the 3D object into a realistic architecture, parameters such as texture and lighting were manipulated. Hence, each architecture was proposed in two colours: a warm reddish one and a cold bluish one. To generate a realistic enlightenment of the of the virtual scene, a specific lighting setting was implemented. The light was a directional one $4 \mathrm{~m}$ high, located $2.5 \mathrm{~m}$ to the right and $4.5 \mathrm{~m}$ before the entrance door outside the architecture. Moreover, the light was inclined towards the door, $18^{\circ}$ around the $x$-axis (width) and $-17^{\circ}$ around the $y$-axis (height), making the lighting assume different shapes depending on architectural factors. In this way, 54 architectures were created to implement a $3 \times 3 \times 3 \times 2$ factorial model with factors SideWalls $\times$ Ceilings $\times$ Windows (each with conditions decreasing, constant, increasing) $x$ Colours (warm, cold). Panel A of Fig. 1 provides a view of the architectural factors from the entrance perspective.

\section{Experimental setup}

The whole experiment was performed in a virtual reality environment realized with the HTC Vive Pro Eye head-mounted display (HMD) to provide a highly immersive experience. It is equipped with two AMOLED screens, with a resolution of $1440 \times 1600$ pixels per eye, a refresh rate of $90 \mathrm{~Hz}$, and a field of view of $110^{\circ}$. Unity was integrated into the HMD via the Steam VR asset to control the experimental procedure and collect data. The experiment ran on a laptop equipped with Windows 10 Home (64-bit), Intel Core i7 $-9750 \mathrm{H}, 32$ GB RAM, and the NVIDIA GeForce RTX 2070 graphics card.

\section{Experimental procedure}

After receiving written instructions about the experimental procedure, the Vive Pro Eye head mounted display (HMD) was placed over the subject's head and arranged in a comfortable way providing a clear view of the virtual environment. Through the movement of a first-person perspective camera, subjects experienced the feeling of walking within the architecture. Specifically, a virtual promenade was realized moving the position of this camera with a speed of $1.25 \mathrm{~m} / \mathrm{s}$ starting from the entrance door and stopping before the third nucleus, applying a smoothing function at the beginning and at the end to prevent motion sickness ${ }^{23}$. Thus, each experimental trial consisted of a virtual promenade $(12.5 \mathrm{~s})$ inside the architecture, followed by the presentation of two consecutive grey panels with a visual analog scale (VAS) where subjects could rate the architectural experience in terms of arousal and valence. With the first scale, subjects answered the question: "This environment makes me feel...", ranging from "Deactivated" to "Activated." With the second scale, subjects answered the question: "This environment provides me ... feelings", ranging from "Unpleasant" to "Pleasant". Subjects used the Vive controller to move the VAS cursor with no time limits to provide their judgments. The 54 architectures were randomly presented, divided into three blocks of 18 architectures each. Blocks were separated by a pause in which subjects were permitted to take the HMD off and have some rest. Panel B of Fig. 1 depicts the timeline of an experimental trial.

\section{Statistical analysis}


Subjective ratings of valence and arousal were analyzed via two distinct $3 \times 3 \times 3 \times 2$ repeated measures ANOVA with SideWalls, Ceiling, Windows (decreasing, constant, increasing) and Colours (warm, cold) as within-subject factors. The correlation between arousal and valence scores was computed by the Pearson correlation coefficient. K-means clustering $(k=2)$ was used to group the environments in the space defined by the arousal and valence dimensions. For each of the four environmental factors we computed the prevalence of the architectures with respect to the experimental condition. Finally, the statistical difference of the observed frequencies distribution was assessed by chi-squared tests. Data analysis was performed with Matlab 2018b (The Mathworks, Inc., Natick, MA, USA) and Statistica 7 (StatSoft Europe) software.

\section{Results}

Results of the rmANOVA on valence ratings are illustrated in Fig. 2. A significant effect was observed for the main factors SideWalls $\left(F(2,56)=29.933, p=1.4 * 10^{-9}, \eta_{p}{ }^{2}=0.516\right)$, Windows $(F(2,56)=4.941, p$ $\left.=.011, \eta_{p}{ }^{2}=0.149\right)$ and Ceiling $\left(F(2,56)=7.135, p=.002, \eta_{p}{ }^{2}=0.203\right)$. Specifically, Bonferroni corrected pairwise comparisons revealed lower valence ratings for those architectures with decreasing side walls distance compared to those with constant $\left(p=6.6^{*} 10^{-6}\right)$ and increasing $\left(p=1.3^{*} 10^{-9}\right)$ one. Increasing windows height also resulted in lower valence scores compared to the constant $(p=.016)$ and decreasing $(p=.044)$ conditions. Finally, architectures with constant ceiling height were associated with higher valence ratings compared to those with increasing $(p=.003)$ and decreasing $(p=.008)$ one. No significant difference among valence scores was observed for the factor Colour $\left(F(1,28)=.075, p=.785, \eta_{p}^{2}=.003\right)$. The interaction SideWalls $x$ Ceiling $\left(F(4,112)=6.219, p=1.5^{\star} 10^{-4}, \eta_{p}{ }^{2}=0.182\right)$ was significant as illustrated in Fig. 3 (Panel A). Bonferroni corrected pairwise comparisons revealed that architectures with increasing side walls distance and constant ceiling height resulted in significantly higher valence scores compared to those architectures with different combinations of side walls distance and ceiling height. Contrarily, those architectures with decreasing side walls distance and increasing ceiling height received significantly lower valence scores compared to those architectures with different combinations of such architectural elements. Also the interaction Windows $x$ Ceiling $\left(F(4,112)=3.297, p=.013, \eta_{p}{ }^{2}=0.105\right)$ returned to be significant as presented in Fig. 3 (Panel B). Specifically, Bonferroni corrected pairwise comparisons revealed that subjects provided significant lower valence scores for those architectures with increasing windows height rather than decreasing $(p=.022)$ or constant $\left(p=5^{\star} 10^{-5}\right)$, but only when the ceiling of the architecture increased between one nucleus and the next one, and not in the decreasing or constant ceiling height condition.

Results of the rm ANOVA on arousal ratings are illustrated in Fig. 4. A significant effect was found for the main factors SideWalls $\left(F(2,56)=20.589, p=1.9 * 10-7, n p^{2}=0.424\right)$ and Windows $(F(2,56)=6.812, p$ $\left.=.002, n p^{2}=0.196\right)$, with a trend for the factor Ceiling $(F(2,56)=2.519, p=.089)$. Specifically, Bonferroni corrected pairwise comparisons revealed that architectures with decreasing side walls distance resulted in higher arousal scores compared to those with constant $(p=2.2 \star 10-4)$ and increasing $\left(p=1.5^{\star} 10-7\right)$ 
side walls distance. Architectures with increasing windows height were perceived as more arousing compared to those with constant $(p=.029)$ and decreasing one $(p=.002)$. No significant difference among the arousal scores was observed for the factor Colour $\left(F(1,28)=.911, p=.348, \eta p^{2}=.031\right)$. Significant interaction of the factor Colour $x$ Ceiling $\left(F(2,56)=4.23, p=.019, n p^{2}=0.131\right)$ is illustrated in Fig. 5, Panel A. Bonferroni corrected pairwise comparison revealed that subjects perceived as more arousing warm architectures rather than cold ones when ceiling decreased between consecutive nuclei ( $p$ $=.008)$. The interaction Windows $x$ Ceiling $\left(F(4,112)=3.747, p=0.007, n p^{2}=0.118\right)$ was also significant (Fig. 5, Panel B), showing that higher arousal scores were collected in architectures with increasing windows height and increasing ceiling, compared to those architectures with different combination of such architectural features.

Results of the cluster analysis are illustrated in Fig. 6 . Architectures are distributed in the space defined by arousal and valence dimensions with a significant and negative correlation $(R=-0.85, p<.001)$. Hence, we applied the $k$-means algorithm $(k=2)$ to segregate the architectures experienced with a high level of arousal and a negative level of valence (HANV) from those experienced with low arousal and positive valence (LAPV). The prevalence of architectures within the two clusters according to their experimental condition can be found in Table 1. We observed that considering the factor SideWalls, architectures were largely unbalanced between the two clusters $\left(X^{2}=20.05, p=1.6 * 10^{-5}\right)$ : the $89 \%$ of architectures with decreasing side walls distance belongs to the HANV cluster, while most of the constant and increasing side walls distance architectures belong to the LAPV cluster (72 and 83\%). Furthermore, we found that the distribution of the architectures within the two clusters according to the ceiling height was close to being significant $\left(\chi^{2}=5.85, p=.054\right)$ : the $78 \%$ of architectures with constant ceiling belong to the LAPV cluster, while the $61 \%$ of architecture with decreasing ceiling belong to the HANV cluster. Clustering architectures according to the factors Windows $\left(\chi^{2}=3.15, p=0.207\right)$ and Colour $\left(\chi^{2}=0.3, p=0.584\right)$ did not return statistically significant results. 
Table 1

Percentage of architecture distribution within the clusters HANV and LAPV according to the experimental conditions.

\begin{tabular}{|c|c|c|c|}
\hline & & HANV & LAPV \\
\hline \multirow[t]{4}{*}{ SideWalls } & Decreasing & 88.89 & 11.11 \\
\hline & Constant & 27.78 & 72.22 \\
\hline & Increasing & 16.67 & 83.33 \\
\hline & & HANV & LAPV \\
\hline \multirow[t]{4}{*}{ Windows } & Decreasing & 38.89 & 61.11 \\
\hline & Constant & 33.33 & 66.67 \\
\hline & Increasing & 61.11 & 38.89 \\
\hline & & HANV & LAPV \\
\hline \multirow[t]{4}{*}{ Ceiling } & Decreasing & 61.11 & 38.89 \\
\hline & Constant & 22.22 & 77.78 \\
\hline & Increasing & 50 & 50 \\
\hline & & HANV & LAPV \\
\hline \multirow[t]{2}{*}{ Colour } & Warm & 48.15 & 51.85 \\
\hline & Cold & 40.74 & 59.26 \\
\hline
\end{tabular}

\section{Discussion}

Virtual reality allowed to emotionally experience a virtual promenade through de-contextualized environments formed by three consecutive nuclei whose architectural features varied progressively. This allowed us to isolate the effects generated by purely architectural factors on subjects' affective states, founding that negative-valenced feelings were generated by narrowing the sidewalls, increasing the windows height or increasing/decreasing the ceiling height. In the first two cases, such modulations also generated a significant increase of the perceived arousal. Results of rmANOVAs and cluster analysis revealed that the architectural feature that more strongly affected valence and arousal ratings was the side walls distance. Finally, we found that our architectural spaces generated either pleasant and low arousing states or unpleasant and high arousing states, possibly arguing that de-contextualized architectural forms may be associated with relaxing or anguishing spaces, and rarely with pleasant and exciting spaces or unpleasant and calming ones. 
Our results showed a preference for the subjects to experience the virtual promenade within wide spaces rather than enclosed ones, arguing that the progressive reduction of the surrounding space was perceived as a constriction, thus leading to uncomfortable states of unpleasantness and high arousal 20,21 . This is in line with enclosure and permeability theories, according to which enclosed spaces, characterized by a reduced possibility to move-through and see-through, are associated with uncomfortable states ${ }^{24}$. Previous studies also revealed that 2D stimuli of enclosed spaces were more likely to generate fear sensation, avoidance decisions and judged as less beautiful relative to open spaces ${ }^{2,3}$. Furthermore, enclosed spaces are associated with situations perceived as less controllable, avoided by human beings because increasing the stress level for their inhabitants ${ }^{13}$. Reasons can be found in survival motivation since enclosed spaces do not typically provide any possible way out ${ }^{24,25}$.

Analogously, the virtual promenade characterized by decreasing ceiling height produced a progressive reduction of the surrounding space thus leading subjects to report unpleasant judgments. We found a preference peak for constant ceiling height of $4 \mathrm{~m}$, resembling results in favour of built environments with $3 \mathrm{~m}$ ceiling height which fosters exploration and visuospatial attention ${ }^{3,4,26}$. Such $1 \mathrm{~m}$ difference with respect to the reported literature could be due to general misperception effects generated by virtual reality 27. In fact, recent findings suggest that egocentric distances in virtual environments are estimated as the $75 \%$ of the modelled virtual distance ${ }^{28}$. Instead, the over-increasing in the height of the ceiling led to perceive the architectural space as less pleasant, as also reported by Baird and colleagues ${ }^{26}$, possibly due to a decreased perception of the spaciousness ${ }^{15}$.

We report that virtual promenades with increasing height of the windows generated unpleasant and high arousing judgments. In such architectural spaces, light penetrated from progressively higher and less accessible points, leading subjects to move towards less enlightened areas and where a possible outdoor view was more difficult to access. Previous research claimed that these aspects are fundamental for the wellbeing of the inhabitants ${ }^{29}$. On the one hand, enabling the sunlight to penetrate the architecture through the windows is essential to promote circadian rhythms, directly affecting human health and wellbeing. Acosta and colleagues ${ }^{6}$ showed that windows size, position, and orientation significantly modulated the amount of sunlight within classrooms, thus varying the circadian stimulus for adolescents. On the other hand, access to outdoor views was found to reduce the stress level at the work office ${ }^{30}$, to provide restoration at home ${ }^{31}$, and benefits to post-operative patients during recovery ${ }^{32}$. Furthermore, the presence of windows is also typically associated with an increased perception of the spaciousness of the environment ${ }^{7,33}$. In line with such findings, our results emphasize that placing the windows closer to the subject height produced a more pleasant experience with lower values of arousal possibly due to an increased perception of the spaciousness $11,34,35$.

The colour modulation of the environments did not affect neither valence nor arousal judgments at the end of the dynamic architectural experience. The specific bluish and reddish colours were selected to generate cold and warm sensations recalling different materials such as concrete and wood, respectively. To deeper investigate how the chromatic aspect of the environment modulates the individual emotional 
state, we need to consider additional chromatic characteristics, such as different value of hue, intensity and saturation.

Our results suggest that modification of forms produces a variation of the extra-personal space which the subject can visually explore and move within, thus affecting the perception of spaciousness. Since it is known that space coding relies on both visual as well as motor circuits ${ }^{36-39}$, we may hypothesize that the emotional experience generated by the dynamic perception of progressive variation of architectural features could exploit the neural circuitry composed by parietal and premotor areas devoted to controlling and planning of voluntary movements ${ }^{40-43}$.

This study demonstrates that virtual reality technologies allow to evaluate emotional perception of people during the progressive variation of architectural features within de-contextualized environments, contributing to foster a human-centered approach to design where mental state of people is fundamental for the creation of novel spaces. This knowledge will assist the design of built settings with features coherent with the social events expected to occur inside them (e.g., collaborative and social interactions in the workplace, patient-staff interaction in healthcare settings, formation of social ties in public spaces). A better understanding of how different architectural features influence and possibly support social interactions will also bring us a step closer to designing for neurodiversity and social sustainability.

\section{Declarations}

\section{Author contribution statement}

P.P, D.R. and G.V. designed the experiment. D.R. provided the materials. P.P. performed data acquisition and analyses. P.P., P.A., F.C., and G.V. interpreted the results. P.P. drafted the manuscript. P.P., P.A., F.C., G.R. and G.V. revised and wrote the manuscript. All the authors have contributed to, seen and approved the final manuscript.

\section{Additional information}

Author G.V. has received research support from Lombardini22. Other authors do not have competing interest.

\section{References}

1. Bower, I., Tucker, R. \& Enticott, P. G. Impact of built environment design on emotion measured via neurophysiological correlates and subjective indicators: A systematic review. Journal of Environmental Psychology, 66, 101344 (2019).

2. Vartanian, O. et al. Impact of contour on aesthetic judgments and approach-avoidance decisions in architecture. PNAS, 110, 10446-10453 (2013).

3. Vartanian, O. et al. Architectural design and the brain: Effects of ceiling height and perceived enclosure on beauty judgments and approach-avoidance decisions. Journal of Environmental 
Psychology, 41, 10-18 (2015).

4. Coburn, A. et al. Psychological and neural responses to architectural interiors. Cortex, 126, 217-241 (2020).

5. Higuera-Trujillo, J. L., Llinares, C. \& Macagno, E. The Cognitive-Emotional Design and Study of Architectural Space: A Scoping Review of Neuroarchitecture and Its Precursor Approaches. Sensors, 21, 2193 (2021).

6. Acosta, I., Campano, M. Ã., Leslie, R. \& Radetsky, L. Daylighting design for healthy environments: Analysis of educational spaces for optimal circadian stimulus. Sol. Energy, 193, 584-596 (2019).

7. Ozdemir, A. The effect of window views openness and naturalness on the perception of rooms spaciousness and brightness: A visual preference study. SRE, 5, 2275-2287 (2010).

8. Palmer, S. E. \& Schloss, K. B. An ecological valence theory of human color preference. PNAS, 107, 8877-8882 (2010).

9. Wilms, L. \& Oberfeld, D. Color and emotion: effects of hue, saturation, and brightness. Psychol. Res, 82, 896-914 (2018).

10. Yildirim, K., Hidayetoglu, M. L. \& Capanoglu, A. Effects of Interior Colors on Mood and Preference: Comparisons of Two Living Rooms. Percept Mot Skills, 112, 509-524 (2011).

11. Kaye, S. M. \& Murray, M. A. Evaluations of an Architectural Space as a Function of Variations in Furniture Arrangement, Furniture Density, and Windows. Hum. Factors, 24, 609-618 (1982).

12. Jalil, N., Ab., Yunus, R. M. \& Said, N. S. Environmental Colour Impact upon Human Behaviour: A Review. Procedia - Social and Behavioral Sciences, 35, 54-62 (2012).

13. Fich, L. B. et al. Can architectural design alter the physiological reaction to psychosocial stress? A virtual TSST experiment. Physiol. Behav, 135, 91-97 (2014).

14. Meyers-Levy, J. \& Zhu, R. The Influence of Ceiling Height: The Effect of Priming on the Type of Processing That People Use. Journal of Consumer Research, 34, 174-186 (2007).

15. Stamps, A. E. Effects of Area, Height, Elongation, and Color on Perceived Spaciousness. Environment and Behavior, 43, 252-273 (2011).

16. Banaei, M., Hatami, J., Yazdanfar, A. \& Gramann, K. Walking through Architectural Spaces: The Impact of Interior Forms on Human Brain Dynamics. Front. Hum. Neurosci.11, (2017).

17. Pasqualini, I., Llobera, J. \& Blanke, O. "Seeing” and “feeling” architecture: how bodily selfconsciousness alters architectonic experience and affects the perception of interiors. Front. Psychol, 4, 354 (2013).

18. Pasqualini, I., Blefari, M. L., Tadi, T., Serino, A. \& Blanke, O. The Architectonic Experience of Body and Space in Augmented Interiors.Front. Psychol.9, (2018).

19. Sanchez-Vives, M. V. \& Slater, M. From presence to consciousness through virtual reality. Nat. Rev. Neurosci, 6, 332-339 (2005).

20. Gallese, V. \& Ruzzon, D. Tuned Architecture. Harmony, Experience and Architecture. overview editore https://www.overvieweditore.com/prodotto/tuned-architecture/ (2016). 
21. Ruzzon, D. Tuning Design (Aracne, 2017).

22. Faul, F., Erdfelder, E., Lang, A. G. \& Buchner, A. G*Power 3: A flexible statistical power analysis program for the social, behavioral, and biomedical sciences. Behav. Res. Methods, 39, 175-191 (2007).

23. Akiduki, H. et al. Visual-vestibular conflict induced by virtual reality in humans. Neurosci. Lett, 340, 197-200 (2003).

24. Stamps, A. E. Effects of Permeability on Perceived Enclosure and Spaciousness. Environment and Behavior, 42, 864-886 (2010).

25. Stamps, A. E. \& Visual Permeability Locomotive Permeability, Safety, and Enclosure. Environment and Behavior, 37, 587-619 (2005).

26. Baird, J. C., Cassidy, B. \& Kurr, J. Room preference as a function of architectural features and user activities. Journal of Applied Psychology, 63, 719-727 (1978).

27. Willemsen, P. \& Gooch, A. A. Perceived egocentric distances in real, image-based, and traditional virtual environments. in Proceedings IEEE Virtual Reality 2002 275-276 (2002). doi:10.1109/VR.2002.996536.

28. Renner, R. S., Velichkovsky, B. M. \& Helmert, J. R. The perception of egocentric distances in virtual environments - A review. ACM Comput. Surv, 46, 1-40 (2013).

29. Nagy, E., Yasunaga, S. \& Kose, S. Japanese office employees' psychological reactions to their underground and above-ground offices. Journal of Environmental Psychology, 15, 123-134 (1995).

30. Leather, P., Pyrgas, M., Beale, D. \& Lawrence, C. Windows in the Workplace: Sunlight, View, and Occupational Stress. Environment and Behavior, 30, 739-762 (1998).

31. Kaplan, R. The Nature of the View from Home: Psychological Benefits. Environment and Behavior, 33, 507-542 (2001).

32. Ulrich, R. S. View through a window may influence recovery from surgery., 224, 420-421 (1984).

33. Moscoso, C. \& Matusiak, B. Aesthetic perception of a small office with different daylighting systems. Indoor and Built Environment, 27, 1187-1202 (2018).

34. Yeom, S., Kim, H., Hong, T., Park, H. S. \& Lee, D. E. An integrated psychological score for occupants based on their perception and emotional response according to the windows' outdoor view size. Building and Environment, 180, 107019 (2020).

35. Yildirim, K., Akalin-Baskaya, A. \& Celebi, M. The effects of window proximity, partition height, and gender on perceptions of open-plan offices. Journal of Environmental Psychology, 27, 154-165 (2007).

36. Berti, A. \& Rizzolatti, G. Coding near and far space. in The cognitive and neural bases of spatial neglect 119-129(Oxford University Press, 2002).

doi:10.1093/acprof:oso/9780198508335.003.0008.

37. Cléry, J., Guipponi, O., Wardak, C. \& Ben Hamed, S. Neuronal bases of peripersonal and extrapersonal spaces, their plasticity and their dynamics: Knowns and unknowns., 70, 313-326 (2015). 
38. Cléry, J., Guipponi, O., Odouard, S., Wardak, C. \& Ben Hamed, S. Cortical networks for encoding near and far space in the non-human primate. Neurolmage, 176, 164-178 (2018).

39. Rizzolatti, G., Fadiga, L., Fogassi, L. \& Gallese, V. The Space Around Us. Science, 277, 190-191 (1997).

40. Jelić, A., Tieri, G., De Matteis, F., Babiloni, F. \& Vecchiato, G. The Enactive Approach to Architectural Experience: A Neurophysiological Perspective on Embodiment, Motivation, and Affordances. Front. Psychol.7, (2016).

41. Kravitz, D. J., Saleem, K. S., Baker, C. I. \& Mishkin, M. A new neural framework for visuospatial processing. Nat Rev Neurosci, 12, 217-230 (2011).

42. Vecchiato, G. et al. Neurophysiological correlates of embodiment and motivational factors during the perception of virtual architectural environments. Cogn. Process, 16, 425-429 (2015).

43. Vecchiato, G. et al. Electroencephalographic correlates of sensorimotor integration and embodiment during the appreciation of virtual architectural environments. Front. Psychol, 6, 1944 (2015).

\section{Figures}

\section{Figure 1}

Panel A: Perspectives of virtual environments for each architectural factor (SideWalls, Ceiling, Windows, Colour) for the corresponding experimental conditions (decreasing, constant, increasing; cold, warm). Panel B: schematic representation of one experimental trial: subject made a virtual promenade between the first two nuclei of the architecture (T0-T1) and then rated the experienced level of arousal and pleasantness (T2). 
A

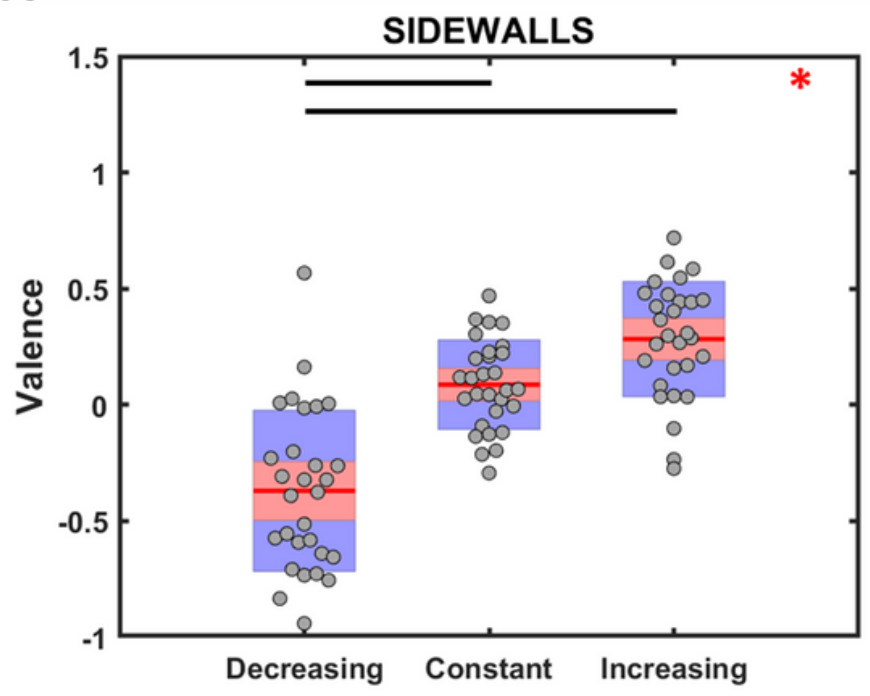

C

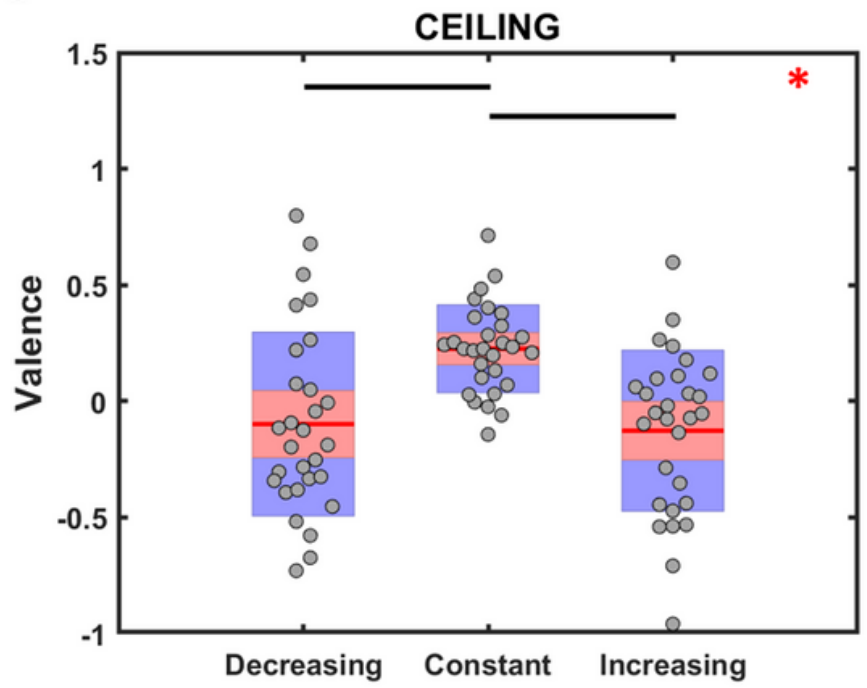

B

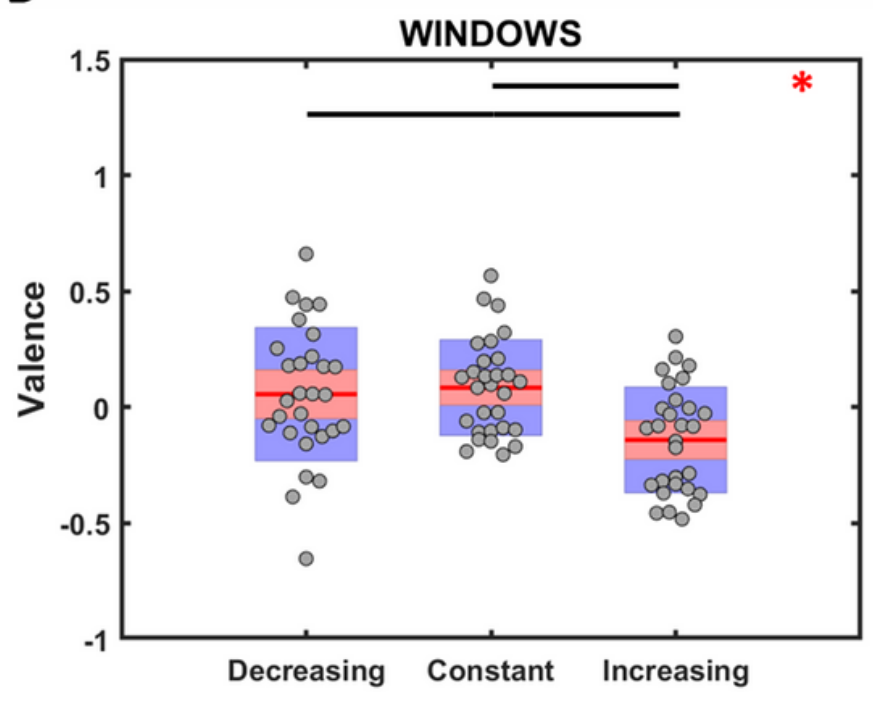

D

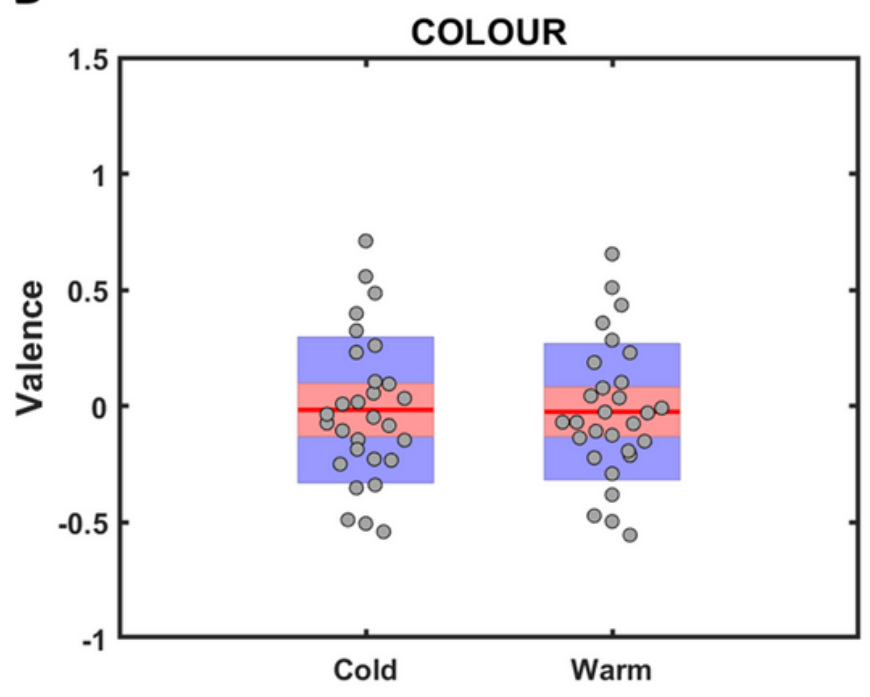

Figure 2

Distribution of the arousal scores for SideWalls, Windows, Ceiling, and Colour are presented in panels A, B, C, D, respectively. Red lines represent the mean value; data are represented as dots laid over a 1.96 standard error mean (95\% confidence interval) in pink and a 1 standard deviation in blue. Red asterisks indicate significant main effect, meanwhile black lines show the significant pairwise comparisons. 
A

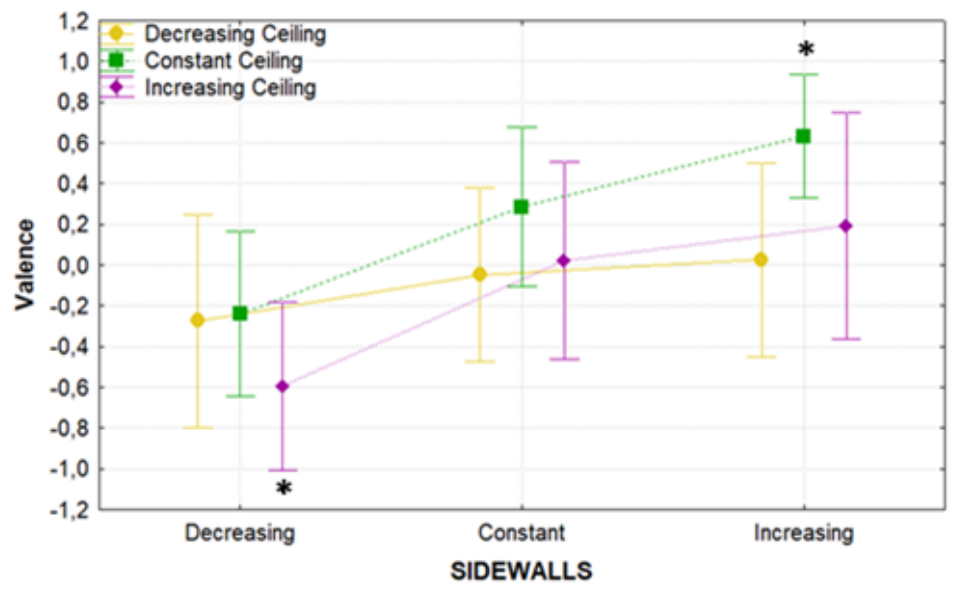

B

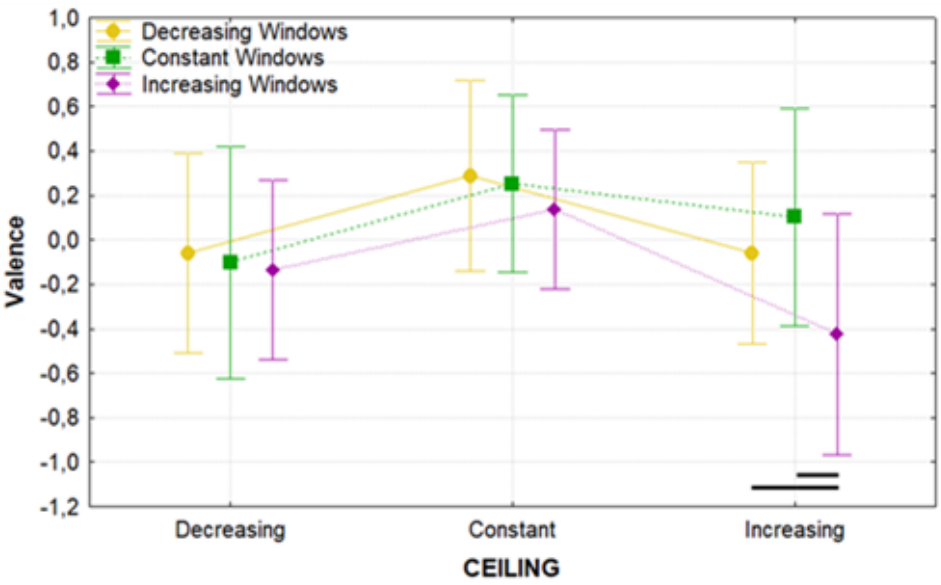

\section{Figure 3}

Panel A: distribution of the valence scores for the two-way interaction SideWalls $x$ Ceiling. Yellow, green and purple lines represent decreasing, constant and increasing ceiling height, respectively. Panel B: distribution of the valence scores for the two-way interaction Ceiling $x$ Windows. Yellow, green and purple lines represent decreasing, constant and increasing windows height, respectively. Data are presented with their mean value with vertical lines representing the $95 \%$ confident interval. Black lines stand for significant pairwise comparison, while black asterisks remark an experimental condition which comparisons with the other conditions are all significant. 
A

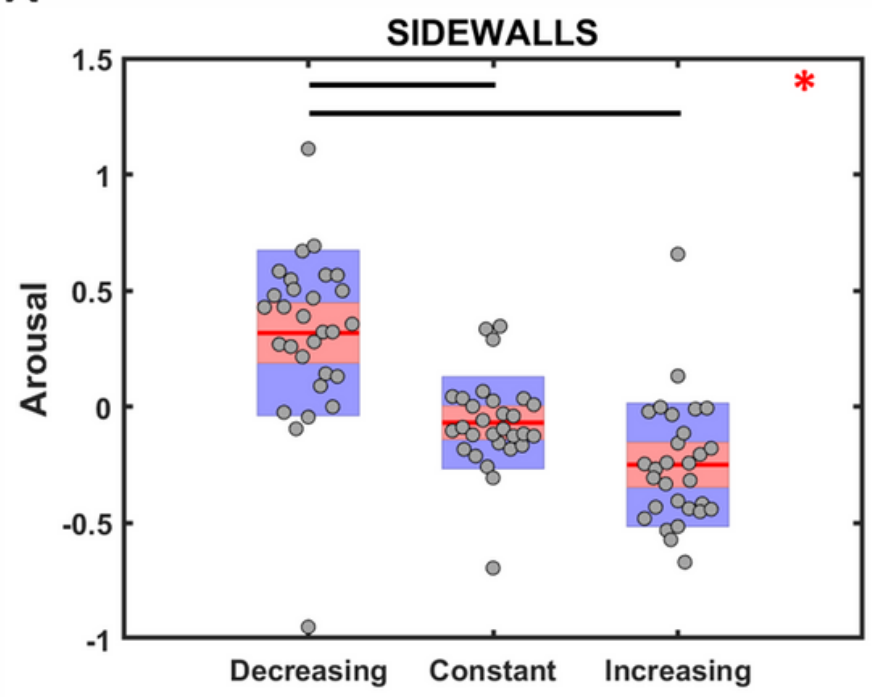

C

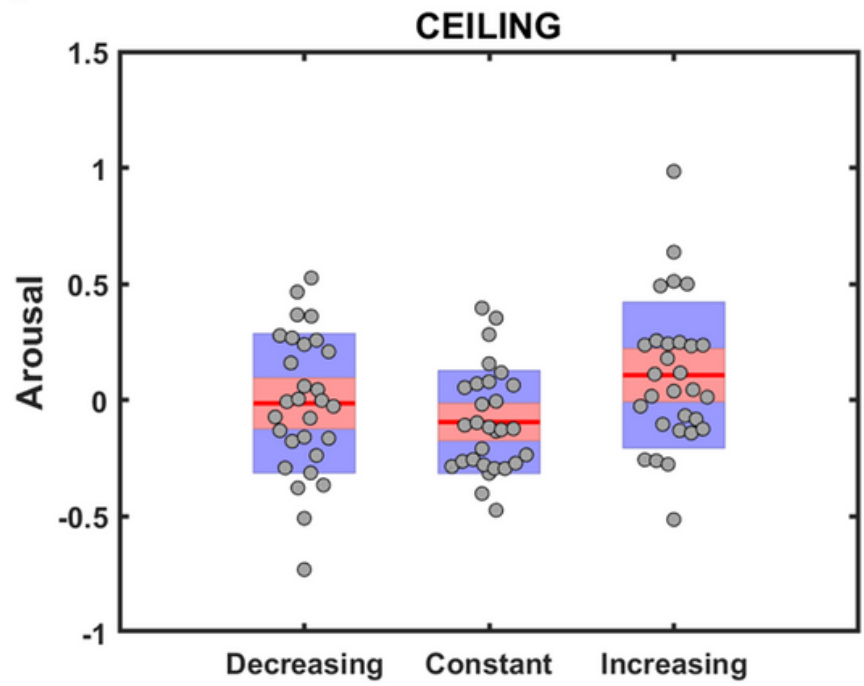

B

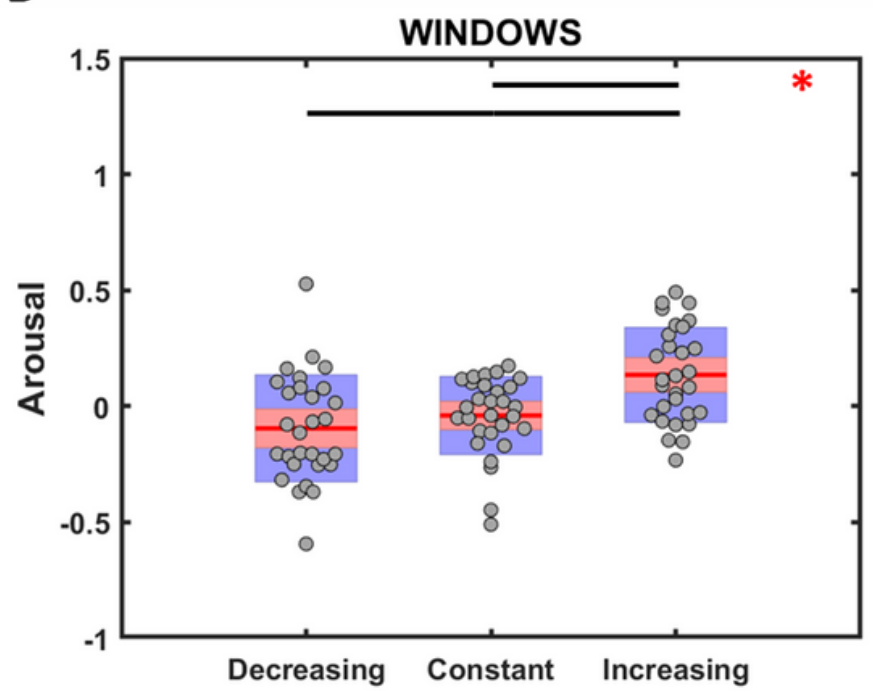

D

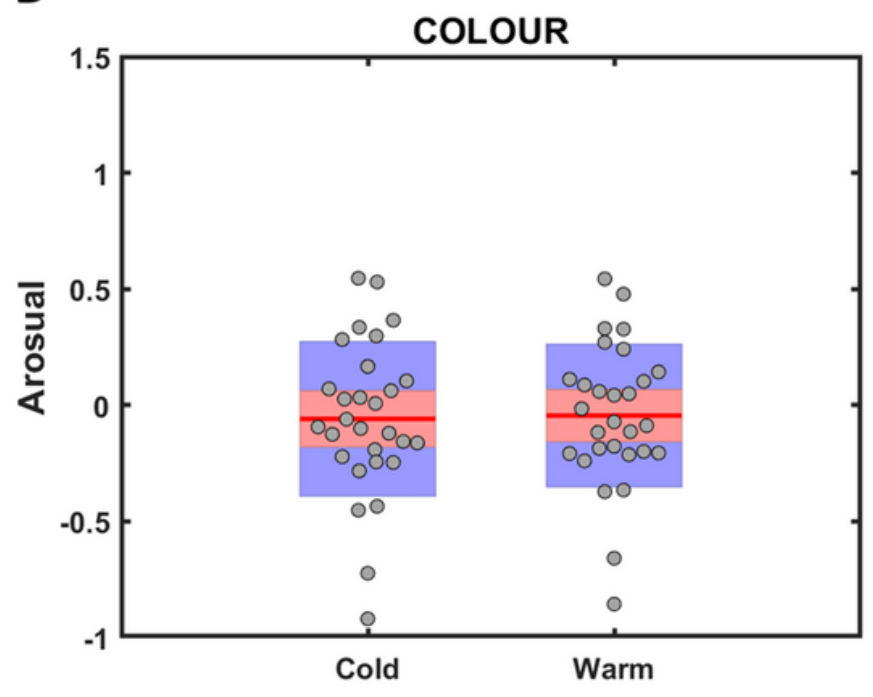

\section{Figure 4}

Distribution of the arousal scores for the main factors SideWalls, Windows, Ceiling and Colour are presented in panels A, B, C, D, respectively. Same colour code as Figure 2.

A

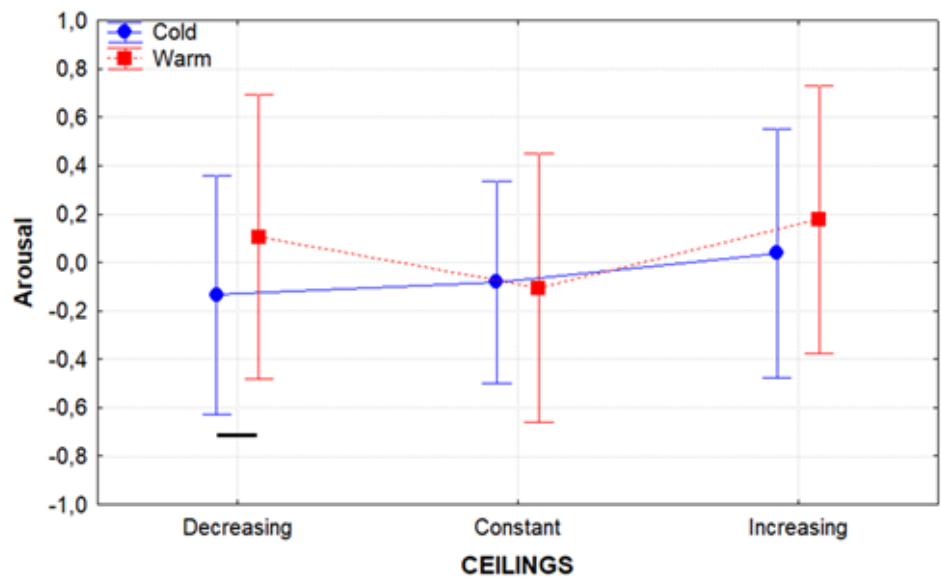

B

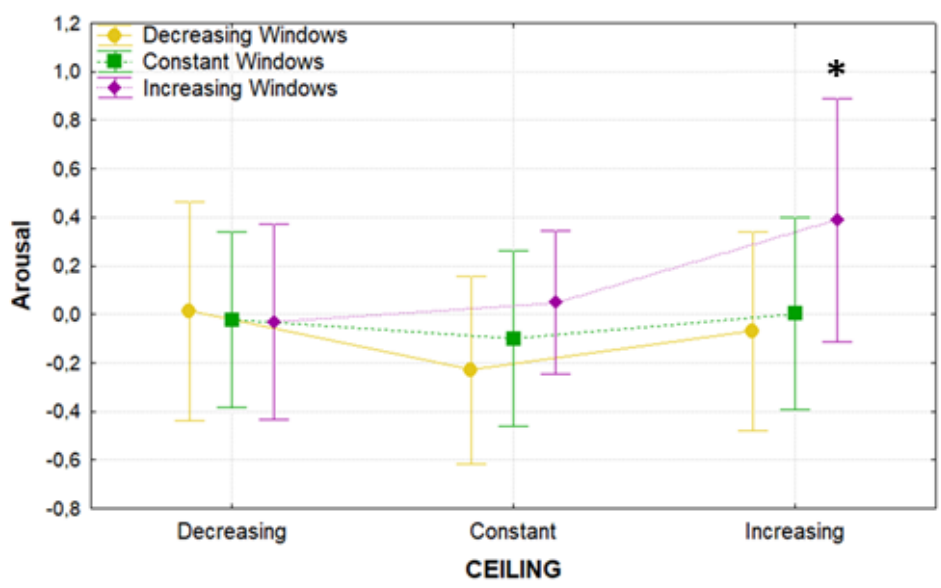




\section{Figure 5}

Panel A: distribution of the arousal scores for the two-way interaction Colour $x$ Ceiling. Blue and red lines represent cold and warm architectures, respectively. Panel B: Distribution of the arousal scores for the two-way interaction Windows x Ceiling, same colour code as Figure 3.

A

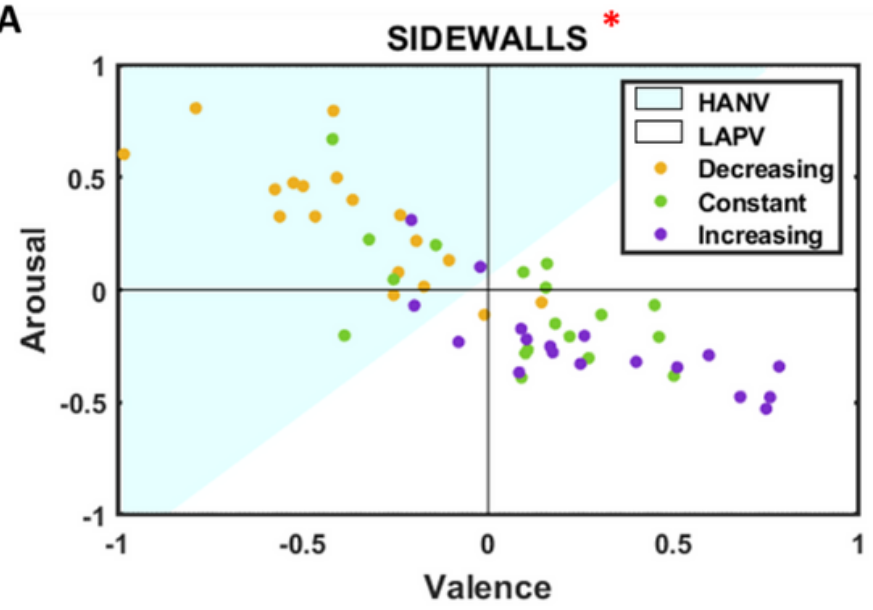

C

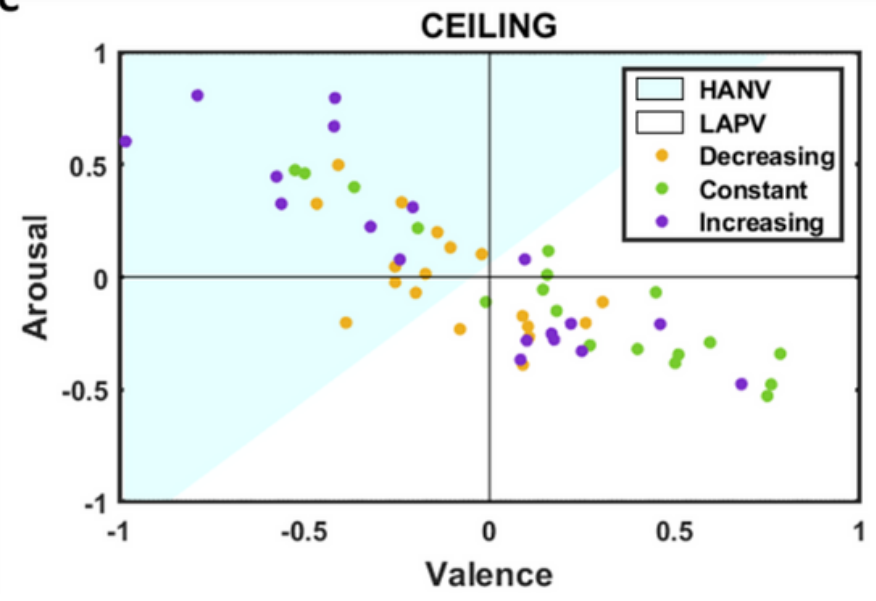

\section{B}

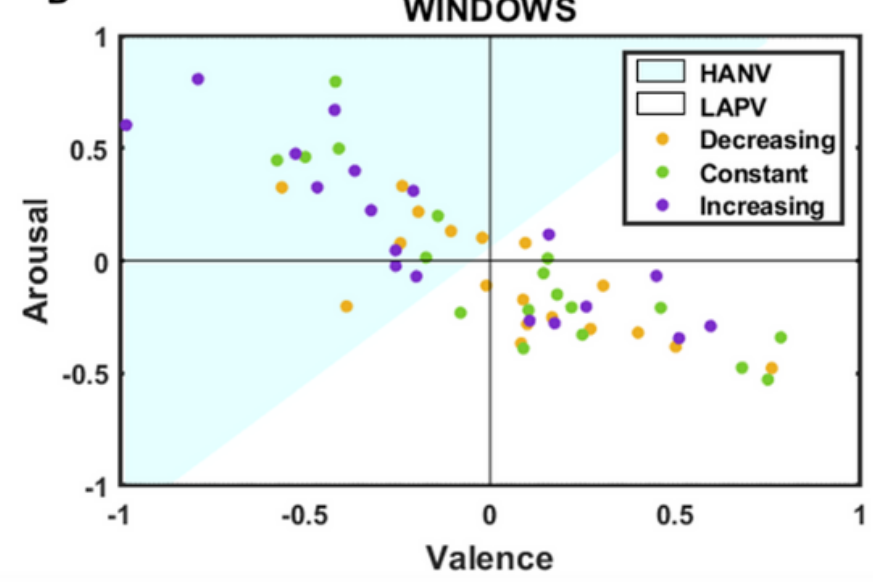

D

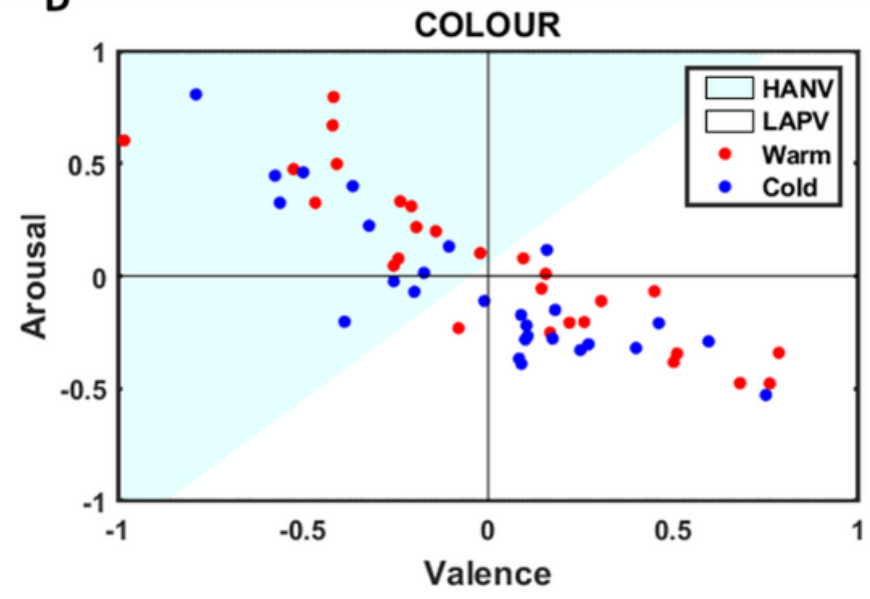

Figure 6

For each panel, the blue section of the plane includes the architectures belonging to the HANV cluster, while the white one comprises architectures within the LAPV cluster. Panel A, B, C: yellow, green, and purple dots identify architectures with decreasing, constant and increasing conditions for SideWalls, Windows, and Ceiling factors, respectively. Panel D: blue and red dots identify architectures with cold and warm texture colour, respectively. The red asterisk indicates statistically significant results. 